testing, and practices around documentation and record retention.

Results (if a Case Study enter NA): Of the 63 responses received, almost all $(95.2 \%, \mathrm{n}=60)$ indicated that their institution defines glucometer critical value thresholds. Of these, the most common threshold for critical-high was $400 \mathrm{mg} / \mathrm{dL}(44.4 \%, \mathrm{n}=28)$ and for critical-low was $50 \mathrm{mg} / \mathrm{dL}(39.7 \%, \mathrm{n}=25)$. A majority $(55.5 \%, \mathrm{n}=35)$ of programs require repeat testing of results that exceeded critical limits. The most popular POC result management software $(50.8 \%, \mathrm{n}=32)$ was RALS (Abbott Diagnostics, Chicago, IL) and the most popular glucometer (56\%, $\mathrm{n}=23$ ) was Roche Accu- Chek Inform II (Roche Diagnostics, Basel, Switzerland). Regarding institutions that disclosed training and competency documentation practices $(93.7 \%, \mathrm{n}=59)$, a majority $(57.6 \%, \mathrm{n}=34)$ used online-only storage, followed by hybrid online- paper storage $(32.2 \%, \mathrm{n}=19)$, and paper-only storage $(10.2 \%$, $\mathrm{n}=6$ ).

Conclusion: Our brief survey has uncovered variations and insights that should raise queries on the feasibility of standardized critical value thresholds, as well as uniform recommendations for retesting critical values. We observed widespread adoption of middleware, as well as online record-keeping. We hope that our findings will trigger further discussions and follow-up studies by other researchers in the POC field.

\section{Vitamin D deficiency Crisis in Egypt}

\section{Abdelmonem, ${ }^{1}$ A. Elhusseny, ${ }^{2}$ H. Wasim, ${ }^{3}$}

M. Shedid, ${ }^{4}$ A.S. Boraik,; ${ }^{5}$ Clinical Laboratory, Stanford

Healthcare - Valleycare, Pleasanton, California, UNITED

STATES; ${ }^{2}$ Port Said University, Port Said, EGYPT;

${ }^{3}$ Meufia University, Shebin El Koum, EGYPT; ${ }^{4}$ El-Zahraa

Laboratory, Benha, EGYPT; ${ }^{5} A L$ NUZHA GENERAL

MEDICAL CENTER, Jeddah, EGYPT

Introduction/Objective: Vitamin D deficiency is a worldwide health problem. Even with the latest medical technology worldwide, vitamin D deficiency is still an ignored epidemic. More than a billion people globally are vitamin D deficient or insufficient. Vitamin D is essential for strong bones as it is responsible for the absorption of calcium, magnesium, and phosphate in the human body. It is essential for building the human immune system and legalizing cell growth. Vitamin D deficiency can result in muscle vulnerability, pain, tiredness, and depression. Severe Vitamin D deficiency can cause Rickets disease.

Objectives: This study aims to predict the percentage of vitamin D deficiency in Egypt.

Methods/Case Report: A total of 498 subjects were enrolled in this study from the Cairo region in Egypt; $203(41 \%)$ were male, $260(52 \%)$ were female, and 35 $(7 \%)$ were children with a median age of 36.2 years, 36.7 years and 5.3 years respectively. A fully automated chemiluminescence immunoassay analyzer (CLIA) was used to measure Vitamin D in all subjects' specimens.

Results (if a Case Study enter NA): The overall prevalence of Vitamin D deficiency was $90.09 \%$. Among the 498 patients, 453 patients $(90.09 \%)$ were Vitamin D deficient (less than $30 \mathrm{ng} / \mathrm{ml}$ ), while 45 patients $(9.03 \%$ ) of the patients were within the normal Vitamin D range. It was observed that 192 patients $(94.54 \%)$ of males, 237 patients $(91.15 \%)$ of females, and 11patients $(31.42 \%)$ of children were Vitamin D deficient. Furthermore, 11 patients $(5.41 \%)$ of males, 23 patients $(8.84 \%)$ of females, and 23 patients $65.71 \%$ of children are within the normal Vitamin D range.

Conclusion: Our findings reveal a significant frequency of vitamin D deficiency in Egypt. Inadequate sun exposure, Insufficient dietary calcium, gastrointestinal disorders, renal diseases, and liver diseases contribute to vitamin D deficiency. We recommend that the Egyptian Health ministry launch an awareness campaign for the severe Vitamin D deficiency in Egypt.

\section{The Utility of PathElective.com as a Curricular Adjunct in Laboratory Medicine Education}

C.M. Lilley, ${ }^{\text {I }}$ M.J. Marin, ${ }^{2}$ J. Wiencek, ${ }^{3}$ L. Fu, K. Wolniak, ${ }^{4}$ C.E. Kanakis, ${ }^{5}$ D. Hermelin, ${ }^{6}$ T. Scordino, ${ }^{7}$ C. Thomas, ${ }^{7}$ K. M. Mirza, ${ }^{8}$ C. Demas ${ }^{9},{ }^{1}$ Stritch School of Medicine, Loyola University Chicago, Chicago, Illinois, UNITED STATES; ${ }^{2}$ Department of Pathology, Immunology and Laboratory Medicine, University of Florida, Gainesville, Florida, UNITED STATES; ${ }^{3}$ Department of Pathology, Microbiology, and Immunology, Vanderbilt University Medical Center, Nashville, Tennessee, UNITED STATES; ${ }^{4}$ Department of Pathology, Northwestern University Feinberg School of Medicine, Chicago, Illinois, UNITED STATES; ${ }_{5}^{5}$ Department of Pathology and Laboratory Medicine, Loyola University Medical Center, Maywood, Illinois, UNITED STATES; ${ }^{6}$ Department of Pathology, Anatomic \& Clinical, Saint Louis University School of Medicine, St. Louis, Missouri, UNITED STATES; ${ }^{7}$ Department of Pathology, University of Oklahoma Medical Center, Oklahoma City, Oklahoma, UNITED STATES;

${ }^{8}$ Department of Pathology and Laboratory Medicine, Loyola University Medical Center, Maywood, Illinois, UNITED STATES; ${ }^{9}$ Department of Pediatrics, University of Michigan, Ann Arbor, Michigan, UNITED STATES

Introduction/Objective: PathElective.com was founded as a means of combating stagnating resident and medical student education due to halting of in-person educational activities during the COVID-19 pandemic. The site has since grown to be included in medical student and resident training programs at numerous institutions across the world, serving as a unique means of delivering highquality and trackable laboratory medicine education. 
Methods/Case Report: The PathElective website was developed using Squarespace, video lectures were recorded by each professor or sourced from openly available web resources incorporating virtual slides, reading assignments, or relevant podcasts at the course director's discretion. Monthly website traffic data were obtained through Squarespace analytics for the first 11 months of the website being available (May 1, 2020, to April 22, 2021). Geographic and source data were obtained through deidentified IP address analysis built-in to Squarespace analytics. Students who registered to take the online courses were assessed before and after interacting with the course materials using a dual form crossover quiz design to prevent memorization of questions and assess comprehension. Quiz data were all anonymous and improvement was determined using a paired t-test. Comparisons between courses were made via percentage improvements.

Results (if a Case Study enter NA): Over the tracking period, PathElective.com received 352,012 page views, 73,550 visits, 33,225 unique visitors, and accrued 5,815 registered users. Most of the visitors (72.8\%) arrived at the site via a direct URL input, $14.9 \%$ through a search engine, and $10.6 \%$ through social media. Most visitors were from the US $(43.8 \%)$ or India $(11.9 \%)$. With a total of 1598 test pairs being analyzed from all clinical pathology modules, the average increase in score was $14.4 \%(95 \%, C I=10.3-$ 18.6, $\mathrm{p}=0.0052)$. All courses experienced a statistically significant increase in scores except for Clinical Chemistry lesson $3.2(7 \%,-1.8-15.9 \% \mathrm{CI}, \mathrm{p}=0.12)$. Courses were well received with a median satisfaction score was very satisfied in all six assessment categories.

Conclusion: PathElective.com is a free and effective means of enhancing clinical pathology training in medical education. Students liked the online format, the quality of the lectures, and course faculty, and felt they could get help if needed. An area of improvement for this platform would be the interaction with students on social media.

\section{Cytogenetics}

\section{A Unique Case of der(16)t(1;16) Identified in B-Lymphoblastic Leukemia}

E. M. Fenu, ${ }^{I}$ D. Lyalin, ${ }^{I}$ G. Insuasti-Belrtran, ${ }^{l}$
M. Beaty, ${ }^{I}$ M. Pettenati, ${ }^{I}$ D. Maracaja ${ }^{I},{ }^{I}$ Pathology,
Wake Forest Baptist Hospital, Winston Salem, North
Carolina, UNITED STATES

Introduction/Objective: A derivative chromosome 16 is rare in hematologic malignancies. There are only two previously reported cases to date, both associated with acute myeloid leukemia $(A M L)$. In these cases, the $t(1 ; 16)$ presented as a der(16)t $(1 ; 16)$ resulting in trisomy $1 \mathrm{q}$. This was the sole anomaly in each case. Cytogenetic abnormalities in B-ALL are common and important for understanding of the pathogenesis, classification and prognosis of the disease. Herein we describe a case of $\operatorname{der}(16) \mathrm{t}(1 ; 16)(\mathrm{q} 12 ; \mathrm{q} 24)$ identified for the first time in a patient with B-cell acute lymphoblastic leukemia (B-ALL), with correlation with morphologic and immunophenotypic findings.

Methods/Case Report: The patient is a 65 year-old male who initially presented with one week of fatigue. A complete blood count showed leukocytosis (white blood cell count of $24.6 \times 103 / \mathrm{uL}$ ), anemia (hemoglobin of $7.9 \mathrm{~g} /$ $\mathrm{dL}$ ), marked thrombocytopenia (platelets of $5 \times 103 / \mathrm{uL}$ ). A differential showed $64 \%$ blasts and peripheral blood flow cytometry confirmed B-lymphoblastic differentiation, with two distinct immunophenotypic populations. The patient's bone marrow biopsy was hypercellular ( $>95 \%$ cellularity) with panhypoplasia and a marked increase in blasts $(88 \%$ by aspirate manual differential). Cytogenetic analysis of the bone marrow also demonstrated the presence of two clonal cell lines. The first cell line was chromosomally normal, while the second had a $\mathrm{t}(9 ; 22)$ translocation and a derived chromosomes 16 from a $\mathrm{t}(1 ; 16)$. FISH analysis confirmed $59 \%$ of cells demonstrated a BCR/ABL1 fusion event.

Results (if a Case Study enter NA): NA

Conclusion: The patient's $\operatorname{der}(16) t(1 ; 16)(\mathrm{q} 12 ; \mathrm{q} 24)$ represents a novel genetic abnormality that has not previously been reported in B-ALL. Although it has been described in other acute leukemias, little is known about this abnormality in B-ALL and its implications in pathogenesis and prognosis. Additional molecular testing, including chromosomal microarray analysis, mate-pair, or long-range DNA sequencing or RNA sequencing, could potentially identify the fusion partners and shed light on pathophysiological mechanisms implicated in the leukemic process.

\section{Cytopathology/Cytology}

\section{Pancreatic Metastasis from Breast Cancer: A Clinicopathological Study of Four Cases by Fine Needle Aspiration}

\author{
M.Y. Chen, ${ }^{I} \quad$ Y. Xu ${ }^{2} ;{ }^{1}$ Medical Scientist Training \\ Program, Baylor College of Medicine, Houston, Texas, \\ UNITED STATES; ${ }^{2}$ Pathology \& Immunology, Baylor \\ College of Medicine, Houston, Texas, UNITED STATES
}

Introduction/Objective: Pancreatic metastases are rare, and the most common neoplasms are carcinomas from the kidney, lung, and breast.

Methods/Case Report: We present four cases of breast ductal carcinoma in patients with metastases to the pancreas diagnosed by endoscopic ultrasound guided fine needle aspiration (EUS-FNA). The four female patients ranged from 37 to 64 years in age. Three patients had 